\title{
O papel da fisioterapia no ambiente hospitalar
}

\author{
The role of physiotherapy in the hospital environment
}

\section{Marcos Vinícius da Conceição Furtado" ${ }^{\bullet}$, Augusto Cezar Ferraz da Costa ${ }^{\circ}$, Jamile Corrêa Silva ${ }^{\bullet}$, Ramon Moraes de Moraes ${ }^{\circ}$}

Escola Superior da Amazônia, Belém, Pará, Brasil. *Autor para correspondência. E-mail: viniifurtado97@gmail.com

Resumo: introdução: A atuação do fisioterapeuta no ambiente hospitalar é desconhecida pela maior parte da população, no entanto a sua presença é indispensável para possibilitar a diminuição dos efeitos deletérios vistos com frequência nos sistemas musculoesquelético e cardiorrespiratório advindos da estadia prolongada dos pacientes no ambiente hospitalar. Este estudo tem como objetivo geral mostrar a atuação do fisioterapeuta no contexto hospitalar, e como objetivo especifico verificar quais técnicas são utilizadas por esses profissionais nas condutas que visam a melhora clínica e funcional dos pacientes. Revisão: No período entre 1973 a 1979 reconheceu-se a importância da fisioterapia nos hospitais, fundamentalmente com a fisioterapia respiratória, o que leva essa época a ser de suma importância para a inserção da fisioterapia respiratória brasileira que, com seu rápido crescimento na década seguinte, consolidou-se como indispensável em todos os hospitais, quando então essa especialidade passou definitivamente a compor também as equipes de terapia intensiva. Sem dúvida, a nova atuação da fisioterapia hospitalar brasileira possibilitou uma importante integração multiprofissional e interdisciplinar, solucionando os acometimentos respiratórios e funcionais adquiridos pelos pacientes que se encontram em cuidados intensivos. Discussão: Os pacientes que se encontram no ambiente hospitalar são suscetíveis a complicações pulmonares e musculoesqueléticas por várias causas, como doenças subjacente, imobilização e infecções. Uma das principais especialidades que promovem a prevenção e o tratamento das fraquezas musculares e doenças respiratórias é a fisioterapia hospitalar. Considerações finais: Podemos concluir, que é de suma importância o fisioterapeuta está inserido na equipe multidisciplinar que compõe o ambiente hospitalar, pois notou-se melhora clínica e funcional após as intervenções fisioterapêuticas serem aplicadas nos pacientes, sendo as medidas com maior impacto positivo a mobilização precoce, aspirações de secreções, monitoramento da ventilação mecânica e reabilitação após cirurgias cardíacas. Além disso, observou-se uma alta hospitalar mais precoce nos pacientes que receberam intervenções fisioterapêuticas.

Palavras-chave: serviço hospitalar de fisioterapia, deambulação precoce, fisioterapia.

\begin{abstract}
The physiotherapist's performance in the hospital environment is unknown to most of the population, however, their presence is indispensable to enable the reduction of the harmful effects frequently seen in the musculoskeletal and cardiorespiratory systems resulting from the prolonged stay of these patients in the hospital environment. This study has the general objective of showing the role of the physiotherapist in the hospital context, and as a specific objective to verify which techniques are used by these professionals in the conducts aimed at the clinical and functional improvement of patients. Review: In the period between 1973 to 1979 the importance of physiotherapy in hospitals was recognized, fundamentally with respiratory physiotherapy, which makes this time more important for the insertion of Brazilian respiratory physiotherapy which, with its rapid growth in the following decade, consolidated itself as indispensable in all hospitals, when that specialty definitely became part of the intensive care teams as well. No doubt, the new role of Brazilian hospital physiotherapy enabled an important multiprofessional and interdisciplinary integration, solving the respiratory and functional impairments acquired by patients in intensive care. Discussion: Patients in the hospital environment are susceptible to pulmonary and musculoskeletal complications from various causes, as underlying diseases, immobilization and infections. One of the main specialties that promote the prevention and treatment of muscle weakness and respiratory diseases is hospital physiotherapy. Final Considerations: We can conclude, which is of great importance the physiotherapist is part of the multidisciplinary team that makes up the hospital environment, because there was a clinical and functional improvement after physical therapy interventions were applied to patients, the measures with the greatest positive impact being early mobilization, secretions aspirations, monitoring of mechanical ventilation and rehabilitation after cardiac surgery. Besides that, an earlier discharge was observed in patients who received physical therapy interventions.
\end{abstract}

Keywords: physical therapy department, hospital, early ambulation, physical therapy specialty. 


\section{Introdução}

No período entre 1973 a 1979 reconheceu-se a importância e o papel da fisioterapia nos hospitais, fundamentalmente com a fisioterapia respiratória, o que leva essa época a ser de suma importância para a inserção da fisioterapia respiratória brasileira na linha de frente aos cuidados de pacientes críticos. Durante o atendimento multiprofissional direcionado aos pacientes que se encontram no centro de terapia intensiva, o fisioterapeuta se faz presente em vários momentos do tratamento intensivo, sendo estes, no atendimento a pacientes que não necessitam de suporte ventilatório invasivo; assistência durante a recuperação pós cirúrgica, com o objetivo de minimizar ou evitar complicações respiratórias, déficit funcionais, e assistência a pacientes críticos que necessitam de intubação orotraqueal, ou seja, que requerem suporte ventilatório invasivo e um elevado monitoramento da equipe (Alves, 2012). Dada a alta complexidade dos procedimentos realizados atualmente pelo Fisioterapeuta especialista em Terapia Intensiva, vistos também o grande número de intercorrências clínicas e admissões que ocorrem durante o período de vinte e quatro horas dentro do ambiente hospitalar, a Associação Brasileira de Fisioterapia Cardiorrespiratória e Fisioterapia em Terapia Intensiva recomenda a presença do fisioterapeuta nas unidades de terapia intensiva adulto, pediátrico e neonatal por um período de vinte e quatro horas ininterruptas, pois observou-se melhora dos indicadores clínicos e financeiros nas unidades cujo o fisioterapeuta se faz presente (ASSOBRAFIR, 2013). Sendo assim, a Portaria GM/MS nº 3432 de 12 de agosto de 1998 estabelece que deve haver para cada 10 leitos, um fisioterapeuta destinado aos cuidados exigidos (Ministério da Saúde, 1998). Na atualidade, observa-se que pacientes gravemente enfermos permanecem por um período prolongado em tratamento intensivo, predispondo a incidência de complicações advindas da imobilidade. Essa imobilidade prolongada possui um grande efeito deletério, com rápida redução da massa muscular e da densidade mineral óssea, havendo também comprometimento em outros sistemas do corpo, sendo essas manifestações notadas já nas primeiras semanas de internação, o que contribui para o declínio funcional e diminuição da qualidade de vida após a alta hospitalar. Nessa vertente, estudos recentes demonstraram que a fraqueza muscular generalizada é uma complicação que acomete de $30 \%$ a $60 \%$ dos pacientes internados nas unidades de terapia intensiva, podendo perdurar de seis meses a até dois anos após a alta, o que acarreta impactos negativos na funcionalidade e qualidade de vida desses indivíduos. Além do mais, pacientes com força muscular periférica reduzida permanecem por mais tempo em ventilação mecânica. Contudo, esses efeitos deletérios do imobilismo podem ser revertidos ou amenizados pela atuação da fisioterapia (Machado et al., 2017).

\section{Revisão}

O objetivo do tratamento fisioterapêutico no ambiente hospitalar é evitar os efeitos deletérios do repouso prolongado no leito, estimular o retorno mais breve às atividades físicas diárias, manter a capacidade funcional, devolver a confiança do paciente, reduzir o impacto psicológico como ansiedade e depressão, reduzir complicações pulmonares, aumentar a oportunidade da alta precoce e promover as bases de um programa domiciliar (Lima et al., 2011). O profissional da Fisioterapia possui permissão por lei (Resolução - COFFITO $n^{\circ} 80 / 87$, art $2^{\circ}$ ) para solicitar exames complementares vinculados à sua atividade profissional, de modo a embasar o diagnóstico fisioterapêutico ou para analisar os impactos da sua conduta no quadro clínico do paciente (COFFITO, 1987). É importante salientar que para se ter resultados eficientes no tratamento fisioterapêutico, deve-se avaliar individualmente as condições clínicas de cada paciente, traçando assim um tratamento adequado ao quadro de cada indivíduo (Cunha et al., 2007). Dessa forma, reuniu-se dez estudos relevantes para demonstrar os impactos das condutas fisioterapêuticas nos pacientes que se encontram no ambiente hospitalar (Tabela 1).

Tabela 1. Apresentação dos estudos selecionados com suas respectivas características e desfechos clínicos.

\begin{tabular}{c|c|c|c|c}
\hline AUTOR/ANO & TÍTULO & MÉTODO & RESULTADOS & CONSIDERAÇÕES FINAIS \\
\hline $\begin{array}{c}\text { Borges et al. } \\
(2009)\end{array}$ & $\begin{array}{c}\text { Fisioterapia motora em } \\
\text { pacientes adultos em terapia } \\
\text { intensiva }\end{array}$ & Revisão de literatura & $\begin{array}{c}\text { A mobilização precoce na unidade de } \\
\text { terapia intensiva é viável e segura, } \\
\text { diminuindo a permanência do } \\
\text { paciente em ventilação mecânica } \\
\text { atividades terapêuticas } \\
\text { progressivas, tais como } \\
\text { exercícios motores na cama, } \\
\text { sedestação a beira do leito, } \\
\text { ortostatismo e transferência } \\
\text { para a cadeira }\end{array}$ \\
\hline & $\begin{array}{c}\text { A importância da atuação do } \\
\text { fisioterapeuta no ambiente } \\
\text { hospitalar }\end{array}$ & Revisão de literatura & $\begin{array}{c}\text { Estudos analisaram o impacto do } \\
\text { fisioterapeuta no ambiente hospitalar } \\
\text { e posteriormente os desfechos } \\
\text { clínicos dos pacientes que receberam } \\
\text { intervenções }\end{array}$ & $\begin{array}{c}\text { As análises demonstraram } \\
\text { achados positivos, como } \\
\text { melhoras clínicas e funcionais } \\
\text { dos pacientes após receberem } \\
\text { intervenções fisioterapêuticas. }\end{array}$ \\
\hline
\end{tabular}




\begin{tabular}{|c|c|c|c|c|}
\hline AUTOR/ANO & TÍTULO & MÉTODO & RESULTADOS & CONSIDERAÇÕES FINAIS \\
\hline $\begin{array}{l}\text { Lima et al. } \\
\quad(2011)\end{array}$ & $\begin{array}{l}\text { Fisioterapia no pós- } \\
\text { operatório de cirurgia } \\
\text { cardíaca: a percepção do } \\
\text { paciente. }\end{array}$ & $\begin{array}{l}\text { Estudo descritivo } \\
\text { transversal, } \\
\text { quantitativo }\end{array}$ & $\begin{array}{c}\text { Pacientes que recebem intervenções } \\
\text { fisioterapêuticas no pré- operatório } \\
\text { adquiriram menos complicações } \\
\text { comparados aos que recebem } \\
\text { somente no pós- operatório }\end{array}$ & $\begin{array}{c}\text { Sob a ótica do paciente, o } \\
\text { tratamento fisioterapêutico } \\
\text { contribui para o sucesso do } \\
\text { processo de reabilitação pós- } \\
\text { cirúrgica. }\end{array}$ \\
\hline $\begin{array}{l}\text { Rotta et al. } \\
\quad(2018)\end{array}$ & \begin{tabular}{|c|} 
Relação entre \\
disponibilidade de serviços \\
de fisioterapia e custos de \\
UTI
\end{tabular} & $\begin{array}{l}\text { Estudo observacional } \\
\text { envolvendo } 815 \\
\text { pacientes em } \\
\text { ventilação mecânica } \\
\text { invasiva por mais de } \\
24 \text { horas }\end{array}$ & $\begin{array}{l}\text { O custo estimado por paciente em } \\
\text { uma primeira internação na UTI é } \\
\text { reduzido quando os serviços de } \\
\text { fisioterapia estão disponíveis o } \\
\text { tempo todo }\end{array}$ & $\begin{array}{c}\text { A disponibilidade dos serviços } \\
\text { de fisioterapia podem reduzir } \\
\text { os custos da UTI e diminuir os } \\
\text { dias de internação hospitalar } \\
\text { dos individuos. }\end{array}$ \\
\hline $\begin{array}{c}\text { Viguria et al. } \\
\text { (2018) }\end{array}$ & $\begin{array}{c}\text { Fisioterapia respiratória em } \\
\text { unidade de terapia } \\
\text { intensiva: revisão } \\
\text { bibliográfica }\end{array}$ & $\begin{array}{l}\text { Revisão bibliográfica } \\
\text { em um período de } 15 \\
\text { anos. }\end{array}$ & $\begin{array}{c}\text { Nos pacientes intubados, as } \\
\text { aspirações , e manobras de } \\
\text { hiperinsuflação manuais são métodos } \\
\text { altamente eficientes para a } \\
\text { prevenção de complicações } \\
\text { respiratórias }\end{array}$ & $\begin{array}{l}\text { Com o fisioterapeuta no } \\
\text { ambiente hospitalar, as } \\
\text { complicações respiratórias } \\
\text { podem ser reduzidas. }\end{array}$ \\
\hline $\begin{array}{l}\text { Zeng et al. } \\
\text { (2017) }\end{array}$ & $\begin{array}{l}\text { Efeito da fisioterapia } \\
\text { respiratória em pacientes } \\
\text { submetidos à ventilação } \\
\text { mecânica: um estudo } \\
\text { prospectivo randomizado } \\
\text { controlado }\end{array}$ & $\begin{array}{l}\text { Estudo prospectivo } \\
\text { randomizado } \\
\text { controlado. }\end{array}$ & $\begin{array}{c}\text { A incidência de pneumonia associada } \\
\text { a ventilação mecânica foi menor no } \\
\text { grupo que recebeu os tratamentos } \\
\text { fisioterapêuticos }\end{array}$ & $\begin{array}{l}\text { Os tratamentos realizados que } \\
\text { visavam a manutenção do } \\
\text { sistema respiratória poderam } \\
\text { reduzir a incidência de } \\
\text { pneumonia associada a } \\
\text { ventilação mecânica. }\end{array}$ \\
\hline $\begin{array}{l}\text { Jerre et al. } \\
\text { (2007). }\end{array}$ & $\begin{array}{r}\text { Fisioterapia r } \\
\text { ventilaçã }\end{array}$ & $\begin{array}{l}\text { Ensaios clínicos e } \\
\text { opiniões de } \\
\text { especialistas }\end{array}$ & $\begin{array}{c}\text { Aspiração traqueal e hiperinsuflação } \\
\text { manual, são algumas das técnicas } \\
\text { utilizadas pelo fisioterapeuta } \\
\text { intensivista }\end{array}$ & $\begin{array}{c}\text { O fisioterapeuta possui } \\
\text { importante participação, } \\
\text { auxiliando na condução da } \\
\text { ventilação mecânica, desde o } \\
\text { preparo e ajuste do ventilador } \\
\text { artificial à intubação. } \\
\end{array}$ \\
\hline $\begin{array}{c}\text { Oliveira et al. } \\
\text { (2013) }\end{array}$ & $\begin{array}{c}\text { Efeitos da técnica expansiva } \\
\text { e incentivador respiratório } \\
\text { na força da musculatura } \\
\text { respiratória em idosos } \\
\text { institucionalizados }\end{array}$ & Estudo longitudinal & $\begin{array}{c}\text { As técnicas associadas como } \\
\text { respiron, descompressão e exercícios } \\
\text { diafragmáticos mostraram grande } \\
\text { eficiência }\end{array}$ & $\begin{array}{c}\text { O estudo apresentou } \\
\text { resultados satisfatórios com a } \\
\text { aplicação de técnicas de } \\
\text { expansão pulmonar, } \\
\text { demonstrando aumento de } \\
\text { força na musculatura } \\
\text { respiratória. }\end{array}$ \\
\hline $\begin{array}{c}\text { Liebano et al. } \\
\text { (2009) }\end{array}$ & \begin{tabular}{|c|} 
Principais manobras \\
cinesioterapeuticas manuais \\
utilizadas na fisioterapia \\
respiratória: descrições das \\
técnicas. \\
\end{tabular} & Revisão de literatura & $\begin{array}{c}\text { Terapias manuais se mostraram } \\
\text { eficientes na eliminação de secreções }\end{array}$ & $\begin{array}{c}\text { Deve-se analisar e avaliar, } \\
\text { quais técnicas são mais } \\
\text { eficientes no tratamento de } \\
\text { determinadas patologias } \\
\text { pulmonares. }\end{array}$ \\
\hline $\begin{array}{c}\text { Tipping et al. } \\
\text { (2016) }\end{array}$ & $\begin{array}{c}\text { Os efeitos da mobilização e } \\
\text { reabilitação ativa na UTI na } \\
\text { mortalidade e na função: } \\
\text { uma revisão sistemática }\end{array}$ & $\begin{array}{l}\text { Uma revisão } \\
\text { sistemática guiada por } \\
\text { lista de verificação } \\
\text { PRISMA e metanálise } \\
\text { de ensaios clínicos } \\
\text { randomizados e } \\
\text { controlados }\end{array}$ & $\begin{array}{c}\text { A metanálise mostrou que a } \\
\text { mobilização e a reabilitação ativas } \\
\text { levaram a maior força muscular na } \\
\text { alta da UTI, maior probabilidade de } \\
\text { caminhar sem assistência, e redução } \\
\text { da mortalidade }\end{array}$ & $\begin{array}{l}\text { A mobilização e a reabilitação } \\
\text { ativa na UTI poderam } \\
\text { melhorar o status da } \\
\text { mobilidade, força muscular e } \\
\text { aumentar dias de vida fora do } \\
\text { hospital. }\end{array}$ \\
\hline
\end{tabular}

Fonte: Furtado et al., 2020.

\section{Discussão}

Em um grande estudo onde analisou-se 109 pacientes após 1 ano de recuperação da síndrome do desconforto respiratório agudo (SDRA), foi verificado que todos os pacientes apresentavam funções físicas pobres, advindas da perda de massa muscular, fraqueza muscular proximal, fadiga, e demonstrou-se que metade dos pacientes nesta coorte (grupo) não puderam retornar aos seus empregos em 1 ano após a alta hospitalar, e as razões relatadas pelos que não retornaram foram desempenho abaixo da média, fadiga constante, fraqueza e status funcional diminuído (Borges et al., 2009). A fisioterapia motora no contexto hospitalar é de fundamental importância, pois através de um estudo conduzido em um hospital de referência, onde incluiu-se na pesquisa 8 pacientes que sofreram acidente vascular encefálico (AVE) que evoluíram com déficit motor, concluiu-se que a fisioterapia empregada duas vezes por dia nesses pacientes foi capaz de reduzir o déficit motor, aumentar a força muscular e devolver a sensibilidade dos membros mais acometidos (Alves, 2012). É notório que a fisioterapia tem assumido papel incontestável no processo de reabilitação cardíaca em sua fase hospitalar, no entanto, pouco se sabe até hoje sobre como o paciente enxerga esse 
processo; sendo pouco investigado a respeito de suas dúvidas, angústias, questionamentos e expectativas diante do tratamento fisioterapêutico, com isso, se torna de vital importância a aplicação de questionários e entrevistas destinados à avaliação do grau de satisfação do indivíduo, ouvindo questões que o mesmo não expõem, como os aspectos que o incomoda ou sobre os momentos desconfortáveis durante a terapia (Lima et al 2011). Em outro estudo clássico onde verificou-se sobre a relação entre a disponibilidade $\mathrm{h}$ / dia dos serviços de fisioterapia e os custos da unidade de terapia intensiva (UTI), pode-se concluir que quanto mais os pacientes recebem fisioterapia durante a primeira internação na unidade de terapia intensiva (UTI), menores são os custos hospitalares e melhores são os prognósticos clínicos (Rotta et al., 2018). É imprescindível oferecer uma assistência fisioterapêutica baseada em evidências, nesse caminho, um estudo importante verificou as evidências das técnicas de expansão pulmonar, tosse, vibração torácica, percussão, drenagem postural, espirometria de incentivo e os sistemas oscilatório e não oscilatório, e pode-se demonstrar que há controvérsias em relação a eficácia de certas técnicas como vibração torácica, percussão, espirometria de incentivo e drenagem postural, precisando assim de estudos mais detalhados para determinar a eficácia das técnicas citadas. No entanto, outro estudo demonstrou que nos pacientes sob ventilação mecânica invasiva, a hiperinsuflação manual e a aspiração de secreções são condutas fisioterapêuticas eficazes para a prevenção de complicações respiratórias (Viguria et al., 2018). Em um renomado estudo randomizado que aplicou no grupo controle intervenções de rotina e no grupo intervenção técnicas fisioterapêuticas como insuflação pulmonar manual, expectoração de vibrações e exercício funcional precoce, sendo avaliados escore fisiológico agudo e avaliação crônica da saúde (APACHE II), índice da relação de oxigenação ( $\mathrm{PaO} 2$ / FiO 2) antes e após o tratamento nos dois grupos, função respiratória, sinais vitais antes e após o tratamento, duração da ventilação mecânica(VM) e tempo de permanência na unidade de terapia intensiva (UTI), pode-se concluir que os melhores desfechos se direcionaram para o grupo intervenção, que apresentou diminuição da incidência de pneumonia associada a ventilação mecânica(PAV), o escore fisiológico agudo e avaliação crônica da saúde (APACHE II) obteve queda importante, e houve aumento do índice de relação de oxigenação ( $\mathrm{PaO} 2$ / FiO 2) no grupo intervenção no decorrer de três dias de tratamento em comparação com o antes do tratamento. Em contrapartida, foram verificados no grupo controle complicações significativas como trombose venosa profunda, atelectasias e dentre outras complicações, afirmando assim que a fisioterapia aumenta as chances de sobrevivência dos indivíduos que se encontram em cuidados intensivos (Zeng et al., 2017). A fisioterapia faz parte do atendimento multidisciplinar oferecido aos pacientes que se encontram na unidade de terapia intensiva (UTI), sua atuação é extensa e se faz presente em vários segmentos do tratamento intensivo e semi-intensivo, impactando de maneira positiva nos desfechos clínicos (Jerre et al., 2007).

\section{Considerações Finais}

Podemos concluir, que os pacientes que se encontram no ambiente hospitalar são suscetíveis a diversas complicações, tais como déficit motor, fraqueza respiratória e baixa funcionalidade. A presença do fisioterapeuta no ambiente hospitalar pode reduzir os impactos negativos nos sistemas musculoesquelético e cardiorrespiratório, através da mobilização precoce, insuflação pulmonar, aspirações de secreções, monitoramento da ventilação mecânica e reabilitação após cirurgias cardíacas. Além disso, foi verificado que as condutas fisioterapêuticas aceleram a alta hospitalar dos pacientes, e possibilitam uma vida mais funcional e independente após a estadia hospitalar.

\section{Agradecimentos}

Agradecemos e dedicamos esta obra a todos os graduandos do curso de Fisioterapia da Escola Superior da Amazônia e a todos os profissionais Fisioterapeutas atuantes nos hospitais do Estado do Pará.

\section{Referências}

Alves, A. N. 2012. A importância da atuação do fisioterapeuta no ambiente hospitalar. Revista Ensaios e Ciências, 1415-6938.

Associação Brasileira de Fisioterapia Cardiorespiratória e Fisioterapa Intensiva [ASSOBRAFIR]. 2013. Parecer $\mathrm{n}^{\mathrm{o}}$ 001/2013. 2013. Assunto: Recomendação de trabalho do Fisioterapeuta no período de vinte e quatro horas em centro de tratamento intensivo. 
Borges, V. M., Oliveira, L. R. C., Peixoto, E., \& Caravalho, N. A. A. 2009. Fisioterapia motora em pacientes adultos em Terapia Intensiva. Revista Brasileira de Terapia Intensiva, 0103-507X.

COFFITO. 1987. Resolução-COFFITO no 80/87, art. $2^{\circ}$.

Cunha, C. S., Toledo, R. V., Nogueira, D. S., \& Januário, B. G. 2007. Atuação da Fisioterapia na Reversão das Atelectasias: Um relato de caso na Unidade de Terapia Intensiva. Cadernos UniFOA, 1982-1816.

Jerre, G., Beraldo, M. A., Silva, T. J., Gastaldi, A., Kondo, C., Leme, F., ... Okamoto, V. 2007. Fisioterapia no paciente sob ventilação mecânica. Revista Brasileira de Terapia Intensiva, 0103-507X

Liebano, R. E., Hassen, A. M., Racy, H. H. M. J., \& Corrêa, J. B. 2009. Principais manobras cinesioterapêuticas manuais utilizadas na fisioterapia respiratória: descrição das técnicas. Revista Ciências Médicas, 18(1), 35-45.

Lima, P. M. B., Cavalcante, H. E. F., Rocha, A. R. M., \& Brito, R. T. F. 2011. Fisioterapia no pós-operatório de cirurgia cardíaca: a percepção do paciente. Revista Brasileira de Cirurgia Cardiovascular, 0102-7638.

Machado, A. S., Neto, R. C. P., Carvalho, M. T. X., Soares, J. C., Cardoso, D. M., \& Alburquerque, I. M. 2017. Effects that passive cycling exercise have on muscle strength, duration of mechanical ventilation, and length of hospital stay in critically ill patients: a randomized clinical Trial. Jornal Brasileiro de Pneumologia, 1806- 3713.

Ministério da Saúde 1998. Portaria GM/MS no 3432. 1998. Estabelece critérios de classificação para as Unidades de Tratamento Intensivo - UTI.

Oliveira, M., Santos, C. L. S., Oliveira, C. F., \& Ribas, D. I. R. 2013. Efeitos da técnica expansiva e incentivador respiratório na força da musculatura respiratória em idosos institucionalizados. Revista Fisioterapia em Movimento, 0103-5150.

Rotta, B. P., Silva, J. M., Fu, C., Goulardins, J. B., Neto, R. C. P., \& Tanaka, C. 2018. Relação entre disponibilidade de serviços de fisioterapia e custos de UTI. Jornal Brasileiro de Pneumologia, 44(3), 184-189.

Tipping, C. J., Harrold, M., Holland, A., Romero, L., Nisbet, T., \& Hodgson, C. 2017. The effects of active mobilisation and rehabilitation in ICU on mortality and function: a systematic review. Intensive Care Medicine, 43(2), 171-183.

Viguria, R. G., Arzoz, E. Y. L., Sola, C., Larraya, A. P., Sangil, P. F., Unamuno, G., \& Berardo, B. M. 2018. Fisioterapia respiratória em la unidade de cuidados intensivos: Revisión bibliográfica. Enfermería intensiva, 29(4), 168-18.

Zeng, H., Zhang, Z., Gong, Y., \& Chen, M. 2017. Effect of respiratory physiotherapy in patients undergoing mechanical ventilation: a prospective randomized controlled Trial. Zhonghua Wei Zhong Bing Ji Jiu Yi Xue, 29(5), 403-406.

\section{Minicurrículo}

Marcos Vinícius da Conceição Furtado. Graduando do $8^{\circ}$ período do curso de Fisioterapia pela Escola Superior da Amazônia (ESAMAZ). Foi monitor por um ano (2019) de Cinesiologia e Biomecânica. Possui estágio extracurricular na UTI do Hospital da Ordem Terceira. Atualmente é Vice-Presidente e Diretor Científico da Liga acadêmica de Fisioterapia em Terapia Intensiva do Estado do Pará (LAFITI).

Augusto Cezar Ferraz da Costa. Possui Graduação em Fisioterapia pelo Centro Universitário do Estado do Pará (2008) (CESUPA); Mestrado Profissional em Ensino em Saúde - Educação Médica(CESUPA); Especialista em Pneumologia pela UNIFESP e atualmente Professor de Fisioterapia Cardiovascular - UTI da Escola Superior da Amazônia; Professor em Fisioterapia Respiratória da FAPEN e Orientador científico da Liga Acadêmica de Fisioterapia em Terapia Intensiva do Estado do Pará (LAFITI).

Jamile Corrêa Silva. Graduanda do $6^{\circ}$ período do curso de Fisioterapia pela Escola Superior da Amazônia (ESAMAZ). Atualmente é monitora de Cinesiologia e Biomecânica, estagiária dos serviços de fisioterapia do Tribunal de Justiça do estado do Pará e Diretora Administrativa da Liga Acadêmica de Fisioterapia em Terapia Intensiva do Estado do Pará (LAFITI). 
Ramon Moraes de Moraes. Graduando do $8^{\circ}$ período do curso de Fisioterapia pela Escola Superior da Amazônia (ESAMAZ). Possui estágio na clínica de Fisioterapia da Escola Superior da Amazônia e atualmente é Diretor Administrativo da Liga Acadêmica de Fisioterapia em Terapia Intensiva do Estado do Pará (LAFITI).

Como citar: Furtado, M.V.C., Costa, A.C.F., Silva, J.C., \& Moraes, R.M. 2020. O papel da fisioterapia no ambiente hospitalar. Pubsaúde, 4, a052. DOI: https://dx.doi.org/10.31533/pubsaude4.a052

Recebido: 6 jul. 2020.

Revisado e aceito: 26 jul. 2020.

Conflito de interesse: os autores declaram, em relação aos produtos e companhias descritos nesse artigo, não ter interesses associativos, comerciais, de propriedade ou financeiros que representem conflito de interesse.

Licenciamento: Este artigo é publicado na modalidade Acesso Aberto sob a licença Creative Commons Atribuição 4.0 (CC-BY 4.0). 